\title{
NON-ALCOHOLIC FATTY LIVER DISEASE: AN UPDATE ON DIAGNOSIS
}

\author{
DAN L. DUMITRASCU ${ }^{1}$, MANUELA G. NEUMAN ${ }^{2,3}$
}

\author{
${ }^{1}$ Iuliu Hatieganu University of Medicine and Pharmacy, Cluj-Napoca, Romania \\ ${ }^{2}$ In Vitro Drug Safety and Biotechnology; Toronto, Ontario, Canada \\ ${ }^{3}$ Department of Pharmacology and Toxicology, Faculty of Medicine, University of \\ Toronto, Toronto, Ontario, Canada
}

\begin{abstract}
Background and aim. The non-alcoholic fatty liver disease (NAFLD) and its sub-entity, the non-alcoholic steatohepatitis (NASH) represent a field of a tremendous progress in recent years. Clinicians need to remain updated with new data on pathogenesis and therapy. The present mini review aims to present some new scientific reports on the diagnosis of NAFLD and NASH for clinical practitioners.

Methods. A systematic literature search of the main international databases was performed. We looked for seminal and innovative papers published in main international languages. A narrative review of the topic was consequently written.

Results. This review describes new data on the diagnosis of NAFLD including NASH. Liver punction biopsy remains the gold standard. However many patients and clinicians prefer to use noninvasive methods. We present the serological tests and the imaging methods used to diagnose inflammation and fibrosis occurring in NAFLD and NASH.

Conclusions. NAFLD-NASH are multifaceted entities that have to be diagnosed and treated by skilled and informed practitioners.
\end{abstract}

Keywords: fibrosis, imaging, non-alcoholic fatty liver, laboratory markers, nonalcoholic steatohepatitis

\section{Introduction}

Non-alcoholic fatty liver disease (NAFLD) is a hepatic manifestation that can be diagnosed by histology, imaging, or both [1]. The patient shows evidence of hepatic fat tissue accumulation in the absence of declared chronic alcohol consumption, or use of drug able to induce steatosis, or hereditary disorders [2]. NAFLD can be histologically distinguished from alcoholic steatohepatitis (ASH) based on absence of alcohol consumption in the history of the patient, and on histological markers: hepatocytes ballooning, lobular inflammation, portal granulocytic inflammation, Mallory-Denk bodies, satellitosis, acute cholestasis, perisinusoidal fibrosis, sclerosing hyaline necrosis, and veno-occlusive disease [3].

The association of NAFLD with the traditional

Manuscript received: 15.12.2017

Accepted: 17.01.2018

Address for correspondence: manuela.neuman@utoronto.ca metabolic conditions: obesity, diabetes mellitus, and dyslipidemia is common. From the morphological point of view, NAFLD is classified into two categories: steatosis and non-alcoholic steatohepatitis (NASH). The morphological examination makes the differentiation. While the biopsy of a patient with simple steatosis shows no hepatocyte ballooning, in NASH the liver puncture biopsy shows steatosis accompanied by chronic inflammation (hepatitis). In this case, the parenchymal cells show ballooning with or without hepatic fibrosis or cirrhosis. These advanced stages may also be associated with several histological changes, including the presence of Mallory's hyaline bodies, and perivenular and perisinusoidal fibrosis [4]. These changes may evolve to fibrosis and cirrhosis [5] mainly in obese persons.

The biochemical examination reflects liver dysfunction [6]. The main changes caused by diabetes are the steatosis and the "pseudo-alcoholic liver disease". Some 
antidiabetic oral drugs may lead to liver injury. Since there is a hepatic injury, NASH lesions have to be distinguishable from the hepatotoxic drug-induced lesions [7].

\section{Diagnosis}

The diagnosis of NAFLD requires the proof of an excessive accumulation of triglycerides in the hepatocytes by imaging or histology methods; simultaneously it is mandatory to exclude any significant alcohol consumption (persons drinking more than 40g/day) [8].

By definition, NASH excludes also any medications (metotrexate), toxicants in environment, parenteral nutrition, Wilson's disease, severe malnutrition, lipodystrophy, and chronic liver disease (hemochromatosis, autoimmune liver disease, chronic viral hepatitis, fatty liver of pregnancy, tyrosinemia) and includes co-morbidities such as metabolic syndrome (MS), obesity, diabetes mellitus) [8].

The gold standard for the diagnosis remains the percutaneous liver biopsy; although not easily accepted by many patients and even by doctors, it offers the most accurate diagnostic criteria.

Histological markers of alcoholic liver disease and NAFLD have similar pathological spectra. They may have variable presentation: simple liver steatosis or the more severe steatohepatitis in the presence of inflammation or even liver cirrhosis when fibrosis progressed in the liver.

Liver biopsy allows the grading (severity of ongoing injury) and staging (degree of progression to cirrhosis) of the liver condition. The criteria for the diagnosis of steatohepatitis include the following: presence of $>5 \%$ macrovesicular steatosis, inflammation, and liver cell ballooning, typically with a predominantly centrilobular distribution. There is also a NAFLD activity score (NAS) which is composed by steatosis, inflammation, and ballooning scores. According to it, we can quantify disease activity. A score of 5 or more is associated with a greater likelihood of having NASH. NAS $<5$ is not able to confirm NASH [9].

The most common findings are high serum triglyceride and low level of HDL in serum. Mild elevations of alanine aminotransferase (ALT) and aspartate aminotransferase (AST), up to 3 fold above the upper limit of normal, if other etiology is absent, may be due to NAFLD. Liver enzyme levels are elevated in NASH patients, with the ALT level generally higher than AST [8].

\section{Inflammatory markers}

In NAFLD, there is an increase in the circulating levels of proinflammatory cytokines and of adipocytokines. Adiponectin, which is an antiinflammatory cytokine, is decreased. The consequence is a disturbance of the proinflammatory and anti-inflammatory balance that leads to hepatocyte necro-inflammation, fibrosis and cellular death. Derivatives of lipid peroxidation may also be involved in the progression of the injuries. Other factors are activated, i.e. profibrogenic cytokines, such as interleukin (IL)-10 and transforming growth factor- $\beta$; or apoptosis favoring factors i.e. serum cytokeratin-18 (CK-18) [10].

Fibrogenesis markers: noninvasive assessment

For the assessment of liver fibrosis without histological examination we can use either biochemical kits of serological tests or imaging methods [10-14].

There are several manners to estimate the fibrosis in the absence of liver puncture biopsy.

One of them, the NAFLD fibrosis score (NAS) can be calculated from the following six measures: age, blood glucose, BMI (body mass index), platelet count, albumin, and AST/ALT ratio.

Another one, BAAT (BMI, age, ALT, triglyceride), is calculated upon these parameters: age, BMI, serum triglycerides, and ALT.

BARD (BMI, AST/ALT ratio, diabetes) is another noninvasive index calculated upon BMI, AST/ALT ratio, diabetes.

A more sophisticated index is the ELF (enhanced liver fibrosis) panel, based on the assessment of the plasma levels of three matrix turnover proteins: hyaluronic acid (HA), tissue inhibitor of metalloproteinase (TIMP-1), and Procollagen III amino terminal peptide (PIIINP).

Hepascore is another one, and requires the measurement of bilirubin, $\gamma$-GTP (gamma-glutamyl transpeptidase), $\alpha 2$-macroglobulin and hyaluronic acid levels.

Hyaluronic acid alone is considered to be a marker of liver fibrosis [11].

FIBROSpect combines hyaluronic acid, TIMP1 and $\alpha 2$-macroglobulin. The test is useful for excluding significant fibrosis and cirrhosis.

Fibrometer requires prothrombin index, platelet count, AST, urea, $\alpha 2$-macroglobulin and hyaluronic acid.

APRI (AST to Platelet Ratio Index) is a simple and easily useful text to evaluate rapidly the liver fibrosis.

NashTest uses an algorithm including 13 parameters: age, sex, height and weight, serum triglycerides, cholesterol, a2-macroglobulin, apolipoprotein A1, haptoglobin, gamma-glutamyl transpeptidase $(\gamma$-GTP), ALT, AST and total bilirubin [12].

\section{Imaging methods for liver firbosis}

The imaging methods are also noninvasive. In the general population and in real life we rely mainly on liver ultrasound or liver enzymes. Most patients are reluctant to accept liver biopsy. Tests that make use of measurement of stiffness as a biomarker of hepatic fibrosis include magnetic resonance elastography and US-based transient elastography [12]. Transient elastography is now fully accepted for assessing fibrosis across a wide spectrum of chronic liver disease [13]. The method is useful also in detecting liver steatosis in diabetic patients [14]. 
Acoustic radiation force impulse (ARFI) shear wave imaging used for the estimation of liver stiffness can be used to differentiate between early and advanced stages of hepatic fibrosis [15].

FibroScan XL probes enable the examination of obese patients [16].

Magnetic resonance is a non-invasive imaging surrogate for the assessment of hepatic steatosis. It can be used to both diagnose and grade NAFLD [17-18].

\section{Other Biomarkers}

The inflammatory markers commonly associated with NAFLD/NASH are: tumor necrosis factor (TNF)- $\alpha$, IL-6, IL- 8, matrix metalloproteinases and high-sensitivity C-reactive protein (hsCRP) $[19,20]$.

The relationship between NAFLD and inflammatory markers such as TNF- $\alpha$, IL- 6 and hsCRP was recently reviewed by Neuman et al. [21].Their levels are increased in obese people compared to lean people. Overweight subjects have a profile similar to obese subjects, in respect to the serum hsCRP levels $[22,23]$.

Apoptosis of the hepatocytes may be estimated via Cytokeratin (CK)-18, the major intermediate filament protein of the liver [24], or by the M30 assay [25].

Hialuronic acid if elevated may predict fibrosis and steatosis [26]. Additionally, hyaluronic acid [11] is included in the ELF panel.

Hepcidin levels are elevated in obese children with NAFLD compared to obese non-NAFLD subjects $(\mathrm{p}<0.001)$. Hepcidin levels were overall comparable between obese patients and controls in a sample of 50 obese patients without NAFLD, 30 obese patients with NAFLD, and 30 non-obese healthy controls [27].

Surdea-Blaga and Dumitraşcu [28] evaluated a possible association between anxiety, depression and NAFLD in 63 patients with normal and elevated serum transaminases. The authors compared scores of total distress, and the presence of symptoms of depression and anxiety. The scores were not statistically different in patients with NAFLD and normal transaminases as compared with patients with NAFLD and elevated transaminases. However, the female gender was prone to depression when compared to the male gender.

A recent article [29] looks into the role of genetics in NAFLD proposing diagnostic markers of NASH, as well as pointing the role of age and ethnic differences in the development of the disease focusing on factors that aggravate the severity. Genetic polymorphisms of ethanol metabolizing enzymes and of CYP 2E1 may influence the evolution of NASH [29].

\section{Conclusions}

The pathogenesis of fatty liver NAFLD/NASH is multifactorial. They are represented by the agents causing the injury, the character of the lesions, the mechanism of liver toxicity and the associated risk factors.

\section{Acknowledgements}

The present article was sponsored by an educational grant from In Vitro Drug Safety and Biotechnology, Toronto, Canada.

\section{References}

1. Chalasani N, Younossi Z, Lavine JE, Diehl AM, Brunt EM, Cusi $\mathrm{K}$, et al. The diagnosis and management of non-alcoholic fatty liver disease: practice Guideline by the American Association for the Study of Liver Diseases, American College of Gastroenterology, and the American Gastroenterological Association. Hepatology. 2012;55:2005-2023.

2. Neuman MG, Voiculescu M, Nanau R, Maor Y, Melzer E, Cohen LB, et al. Non-alcoholic steatohepatitis: Clinical and translational research. J Pharm Pharm Sci. 2016;19(1):8-24.

3. Tannapfel A, Denk H, Dienes HP, Langner C, Schirmacher P, Trauner M, et al. Histopathological diagnosis ofnon-alcoholic and alcoholic fatty liver disease. Virchows Arch 2011;458:511-523.

4. Neuman MG, French SW, French BA, Seitz HK, Cohen LB, Mueller S, et al. Alcoholic and non-alcoholic steatohepatitis. Exp Mol Pathol. 2014;97:492-510.

5. Adler M, Schaffner F. Fatty liver hepatitis and cirrhosis in obese patients. Am J Med. 1979;67:811-816.

6. Ludwig J, Viggiano TR, McGill DB, Oh BJ. Nonalcoholic steatohepatitis: Mayo Clinic experience with a hitherto unnamed disease. Mayo Clin Proc. 1980;55:434-438.

7. Zimmerman HJ, Ishak KG. Non-alcoholic steatohepatitis and other forms of pseudoalcoholic liver disease. In: Hall P (Ed). Alcoholic Liver Disease Pathology and Pathogenesis. 2nd Ed. London: Edwarde Arnold, 1995, 165-198.

8. Neuman MG, Malnick S, Maor Y, Nanau RM, Melzer E, Ferenci P, et al. Alcoholic liver disease: Clinical and translational research. Exp Mol Pathol. 2015;99(3):596-610.

9. Brunt EM, Janney CG, Di Bisceglie AM, Neuschwander-Tetri BA, Bacon BR. Nonalcoholic steatohepatitis: a proposal for grading and staging the histological lesions. Am J Gastroenterol. 1999;94(9):2467-2474.

10. Angulo P, Hui JM, Marchesini G, Bugianesi E, George J, Farrell GC, et al. The NAFLD fibrosis score: a noninvasive system that identifies liver fibrosis in patients with NAFLD. Hepatology. 2007; $45: 846-854$.

11. Neuman MG, Cohen LB, Nanau RM. Hyaluronic acid as a non-invasive biomarker of liver fibrosis. Clin Biochem. 2016;49(3):302-315.

12. Poynard T, Ratziu V, Charlotte F, Messous D, Munteanu M, Imbert-Bismut F, et al. Diagnostic value of biochemical markers (NashTest) for the prediction of non alcoholo steato hepatitis in patients with non-alcoholic fatty liver disease. BMC Gastroenterol. 2006 Nov 10; 6:34.

13. Neuman MG, Cohen LB, Nanau RM. Biomarkers in nonalcoholic fatty liver disease. Can J Gastroenterol Hepatol. 2014;28 (11):607-618.

14. Sporea I, Mare R, Lupuşoru R, Sima A, Sirli R, Popescu A, et al. Liver Stiffness Evaluation by Transient Elastography in Type 2 Diabetes Mellitus Patients with Ultrasound-proven Steatosis. J Gastrointestin Liver Dis. 2016;25(2):167-174.

15. Palmeri ML, Wang MH, Rouze NC, Abdelmalek MF, Guy $\mathrm{CD}$, Moser B, et al. Noninvasive evaluation of hepatic fibrosis 
using acoustic radiation force-based shear stiffness in patients with nonalcoholic fatty liver disease. J Hepatol. 2011;55:666-672. 16. Loomba R, Wolfson T, Ang B, Hooker J, Behling C, Peterson $\mathrm{M}$ et al. Magnetic resonance elastography predicts advanced fibrosis in patients with nonalcoholic fatty liver disease: A prospective study. Hepatology. 2014;60(6):1920-1928.

17. Loomba R, Wolfsen T, Haufe W, Hooker J, Szeverneyi N, Ang $\mathrm{B}$ et al. Novel 3-dimensional magnetic resonance elastography accurately diagnoses nonalcoholic steatohepatitis and advanced fibrosis in patients with biopsy-proven

nonalcoholic fatty liver disease: A prospective cohort study. J Hepatol 2014;60(1):S33.

18. Myers RP, Pomier-Layrargues G, Kirsch R, Pollett A, DuarteRojo A, Wong D, et al. Feasibility and diagnostic performance of the FibroScan XL probe for liver stiffness measurement in overweight and obese patients. Hepatology. 2012;55:199-208.

19. Pacifico L, Bezzi M, Lombardo CV, Romaggioli S, Ferraro F, Bascetta $\mathrm{S}$, et al. Adipokines and $\mathrm{C}$-reactive protein in relation to bone mineralization in pediatric nonalcoholic fatty liver disease. World J Gastroenterol. 2013;19:4007-4014.

20. de Piano A, de Mello MT, Sanches Pde L, da Silva PL, Campos RM, Carnier J, et al. Long-term effects of aerobic plus resistance training on the adipokines and neuropeptides in nonalcoholic fatty liver disease obese adolescents. Eur J Gastroenterol Hepatol 2012;24:1313-1324.

21. Neuman MG. Cytokines and chemokines in liver disease. In: Al-Mahtab M, Rahman S (Eds). Liver, a complete book on Hepato-Pancreato-Biliary Diseases. India: Elsevier Reed India Ltd, 2009, pp 19-24.

22. Kitsios K, Papadopoulou M, Kosta K, Kadoglou N, Papagianni M, Tsiroukidou K. High-sensitivity C-reactive protein levels and metabolic disorders in obese and overweight children and adolescents. J Clin Res Pediatr Endocrinol. 2013;5:44-49.

23. Alkhouri N, Carter-Kent C, Lopez R, Rosenberg WM, Pinzani $\mathrm{M}$, Bedogni $\mathrm{G}$, et al. A combination of the pediatric NAFLD fibrosis index and enhanced liver fibrosis test identifies children with fibrosis. Clin Gastroenterol Hepatol. 2011;9:150-155.

24. Shen J, Chan HL, Wong GL, Chan AW, Choi PC, Chan HY, et al. Assessment of non-alcoholic fatty liver disease using serum total cell death and apoptosis markers. Aliment Pharmacol Ther. 2012;36:1057-1066.

25. Toyoda H, Kumada T, Kiriyama S, Tanikawa M, Hisanaga Y, Kanamori A, et al. Higher hepatic gene expression and serum levels of matrix metalloproteinase-2 are associated with steatohepatitis in non-alcoholic fatty liver diseases. Biomarkers. 2013;18:82-87.

26. Demircioğlu F, Görünmez G, Dağıstan E, Göksügür SB, Bekdaş M, Tosun M, et al. Serum hepcidin levels and iron metabolism in obese children with and without fatty liver: casecontrol study. Eur J Pediatr. 2014;173:947-951.

27. Puri K, Nobili V, Melville K, Corte CD, Sartorelli MR, Lopez R, et al. Serum bilirubin level is inversely associated with nonalcoholic steatohepatitis in children. J Pediatr Gastroenterol Nutr. 2013;57:114-118.

28. Surdea-Blaga T, Dumitraşcu DL. Depression and anxiety in nonalcoholic steatohepatitis: is there any association? Rom J Intern Med. 2011;49(4):273-280.

29. Neuman MG, French SW, Zakhari S, Malnick S, Seitz HK, Cohen LB, et al. Alcohol, microbiome, life style influence alcohol and non-alcoholic organ damage. Exp Mol Pathol. 2017;102(1):162-180. 